\title{
Test Instrumentation for a Laser Scanning Localization Technique for Analysis of High Speed DRAM devices
}

\author{
Martin Versen, Achim Schramm, Jan Schnepp \\ Qimonda AG, Am Campeon 1-12, D-85579 Neubiberg, Germany \\ Dorina Diaconescu \\ Infineon AG, Otto-Hahn-Ring 6, D-81739 München, Germany
}

\begin{abstract}
Soft defect localization (SDL) is a method of laser scanning microscopy that utilizes the changing pass/fail behavior of an integrated circuit under test and temperature influence. Historically the pass and fail states are evaluated by a tester that leads to long and impracticable measurement times for dynamic random access memories (DRAM). The new method using a high speed comparison device allows SDL image acquisition times of a few minutes and a localization of functional DRAM fails that are caused by defects in the DRAM periphery that has not been possible before. This new method speeds up significantly the turn-around time in the failure analysis (FA) process compared to knowledge based FA.
\end{abstract}

\section{Introduction}

Standard methodologies for laser scanning localization technique are shown in Fig. 1 and 2. The methods in Fig. 1 are known as TIVA (Thermally Induced Voltage Alteration) and OBIRCH (Optical Beam Induced Resistance Change): a constant current or voltage source is applied to the device under test (DUT) and a voltage or current drop or increase is measured at the device, respectively [1-4].

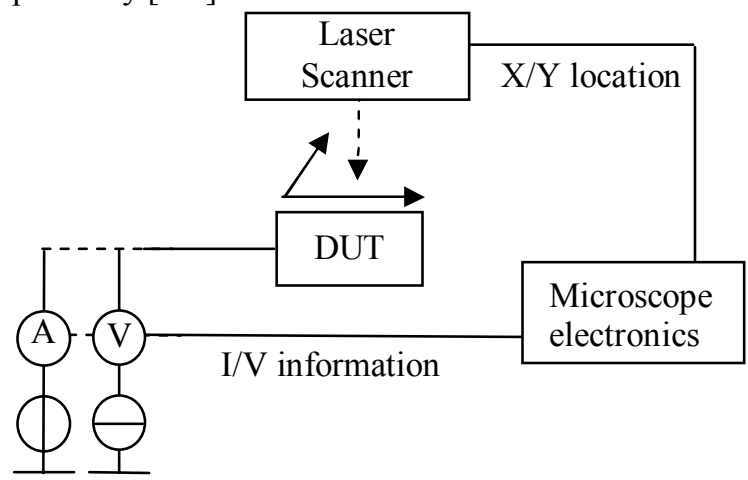

Figure 1: Laser scanning localization setup with analog input.
Both methods use an analogue input of the microscope electronics. The input is valid basically throughout the complete pixel dwell time, the time that is needed to measure one location on the DUT. The disadvantage is that local defects within a DRAM cannot be localized as the interface and output does not change in case of an array defect. For most problems, bitmapping of DRAM array related failures and TIVA and OBIRCH for power net problems in the periphery, the tool set is complete.

Nevertheless, functional failures that are caused by soft defects in the periphery cannot be analyzed as they have neither array specific addresses for bitmapping nor a VDD dependency for TIVA or OBIRCH. Soft defect localization (SDL) methods allow a localization of functional fails within the periphery of the DRAM that are not accessible with any bitmap. Laser scanning localization with a test system utilizes a synchronous input of the microscope electronics shown in Fig. 2: a functional pattern is executed and any local defect may cause a fail depending on the pattern. The tester evaluates the pass or fail result from the DUT and sends a digital signal together with a trigger to the microscope electronics.

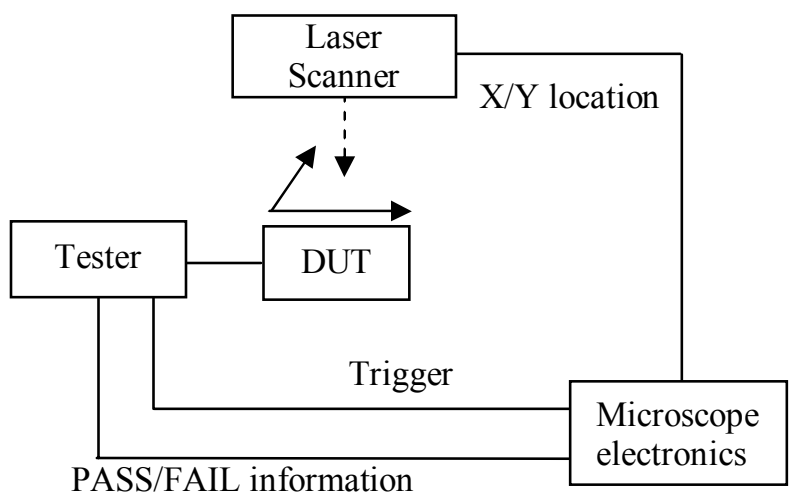

Figure 2: Laser scanning localization setup with synchronous input. 
This technique allows the localization of any temperature dependent defect that causes any function failure. The drawback is clearly that the pattern runtime can be much longer than the minimum pixel dwell time. And even if the pattern is short, the test-to-test index time can be longer than the pixel dwell time. The consequence is that the pixel dwell time must be adjusted. There are some modifications to this method to improve the signal to noise ratio. One example is the use of a lock-in assisted SDL [5], but unfortunately standard and the improved methods can not be used for DRAMs, because there are no scan chains and the operation frequencies are in $>100 \mathrm{Mhz}$ range.

Nevertheless there has been an attempt to utilize the laser scanning methods for DRAMs [6]. Fig. 3 shows an experimental configuration, in which a DRAM output is directly connected to the microscope electronics using again its analog input. The DRAM is operating at 100 $\mathrm{MHz}$ and the data speed is $200 \mathrm{Mb} / \mathrm{s}$. As the executed pattern is a continuous Read, the output becomes quasistatic. The PASS/FAIL information is implicitly encoded in the data out information. The usage is only limited as any interruption of the continuous read, thus any modification of the pattern, changes the measurable signal. So this method is anything but generally applicable.

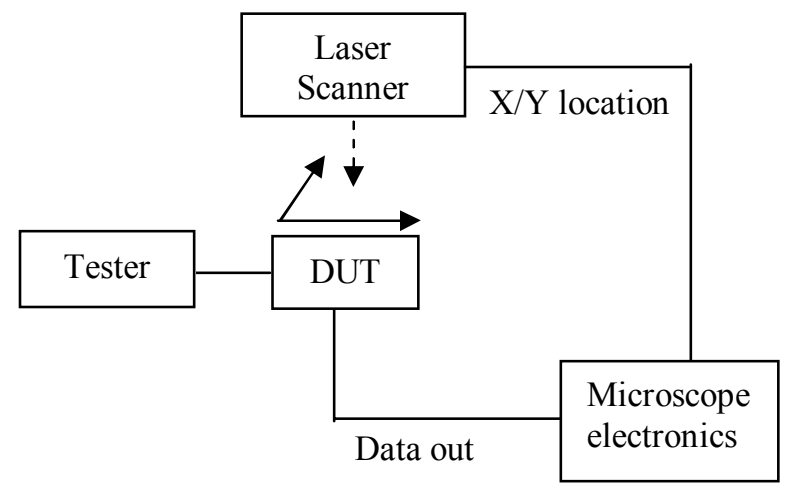

Figure 3: Laser scanning localization setup with analog input, but tester excitation.

The newly proposed methodology is depicted in Fig. 4. It is a laser scanning localization technique similar to the standard synchronous technique with the exception that the tester only drives the DUT. The single bit comparator (SBC) compares the data received with previously programmed data values and sends either a low or a high signal pulse (the PASS/FAIL signal form before) to the analog input of the microscope, which corresponds to the actual qualification. The advantages are a data speed of up to $200 \mathrm{Mb} / \mathrm{s}$ and an adjustable pulse length for the PASS/FAIL signal that can be changed according to both the pattern run time and the pixel dwell time.

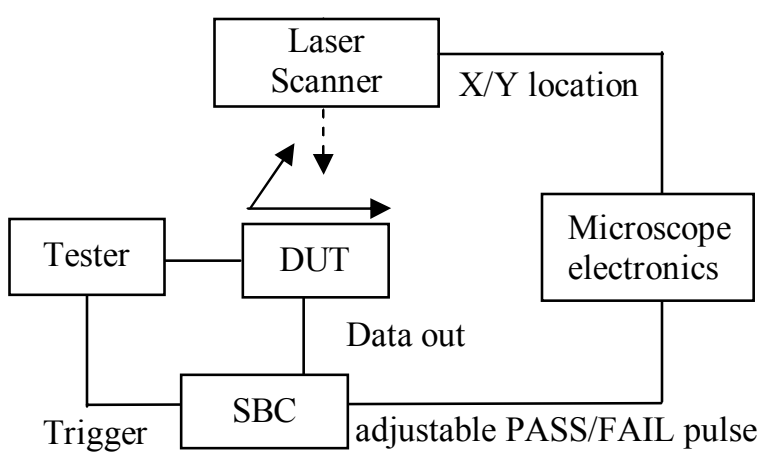

Figure 4: Laser scanning localization setup with the new instrumentation, the Single Bit Comparator (SBC).

The next part of this paper describes the functional blocks of the SBC, while the third part gives some timing characteristics of the SBC. The fourth part shows the successful demonstration and the application of the new tool in the Failure Analysis (FA) lab.

\section{Single Bit Comparator Instrument}

A functional block diagram of the single bit comparator is shown in Fig. 5. The SBC consists of two CPLDs (Complex Programmable Logic Devices) called CPLD_1 and CPLD_2, an 8051 microcontroller, a display and obligatory peripheral circuitry.

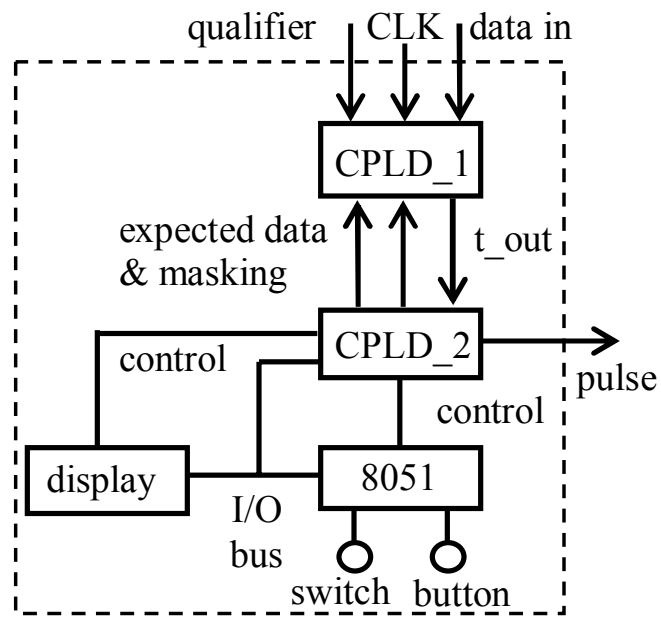

Figure 5: Block diagram of the single bit comparator.

The microcontroller forms together with the display, a rocker-switch and a button the user interface. The user interface allows to define the expected data and an associated bit-mask. Furthermore the user may define the width of the output pulse controlling the microscope.

CPLD_2 acts as "glue logic" to connect all the other devices. In particular it latches the expected data and the bit mask and applies them as parallel 8 bit words to 
CPLD_1. Additionally CPLD_2 drives the output pulse, which controls the input of the microscope.

CPLD_1 receives the same clock as the DRAM (driven by the tester), a qualifier (generated by the tester, active during read bursts) and a data-in line driven by the DQ of the DRAM. When the qualifier is active CPLD_1 takes the serial data burst on the data-in line and compares it to the expected data. The bit-mask allows to restrict the comparison to arbitrary bits (burst positions). Whenever a match is identified the t_out signal to CPLD_2 gets activated. CPLD_2 "stretches" this pulse by means of an internal counter and generates the desired output pulse of defined width to control the microscope.

For CPLD 1 a Xilinx Coolrunner II has been chosen, as this CPLD type works on rising and trailing clock edge making it ideal to receive DDR data. Furthermore this CPLD type allows to change easily the IO-voltage from $1.5 \mathrm{~V}$ to $3.3 \mathrm{~V}$. As the IO-voltage for CPLD_1 is derived from the DRAMs supply voltage, the CPLDs work as level translators between the DRAM / tester on one side and the microcontroller / microscope on the other side. Therefore the SBC can be used without reprogramming or other complications for DDR1, DDR2 and DDR3. CPLD_2 has then been taken from the same CPLD type for simplicity reasons and as it fitted easily to the right performance / complexity class.

\section{Verification of the SBC}

The SBC functionality is verified by use of an IMS bench test system that is also used as a test system for the DRAM in the fourth part. The IMS tester acts here simply as a very convenient waveform generator. Each channel is also connected to a TEKTRONIX TDS 784A oscilloscope for the timing measurements.

The waveforms and the timing definitions for the incoming data and the qualifier that validate the data are shown in Fig. 6. The CLK is running at a rate of 10ns or a frequency of $100 \mathrm{MHz}$. The data waveforms have a data width of $5 \mathrm{~ns}$. As the inputs of CPLD_1 are not synchronous, the edge placement has to be measured with a simple 01010101 pattern and the use of the masking bit. In the case of the first burst bit, all the bits are masked except the first burst bit. The same applies for the measurement for the last burst bit: all bits are masked except the last one. The timings for the data setup and data hold times for the first and the last burst bit are measured with the oscilloscope's cursor functions and are included in Fig. 6. The timings do vary and this has to be taken into account for validating the data in the application. The tester edge accuracy is better than $250 \mathrm{ps}$ so only $t_{\mathrm{DH} 2}$ is possibly critical. Consequently the qualifier placement has to be done carefully in the application for either a PASS reference DUT or in the PASS state of the failing DUT.
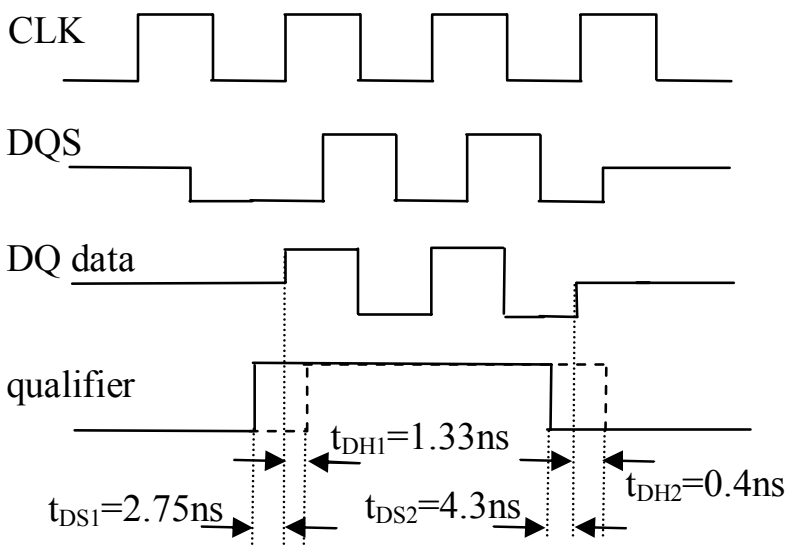

Figure 6: Waveforms of the incoming CLK, data and qualifier including the data setup and hold timings for the qualifier.

\section{Application}

The laser scanning localization of the defect on the DRAM-device is done with a Hamamatsu laser scanning microscope PHEMOS 1000 after a backside preparation of the die. The DUT is scanned with a $1.3 \mu \mathrm{m}$ laser beam which induces a local heating but no carrier generation. The reflected light can be used as input of the imaging system and than an optical image of the die is obtained. On the other side, using the pulse generated by the SBC as input, the DUT response to the laser beam position can be mapped as an electrical image. A heat sensitive defect will result in a spot in this image. The optical and electrical images can be overlaid for an easier identification of the fault location. One of the DRAM data lines is used as the input to the SBC as it is shown in Fig. 4 and the pulse from the SBC is the input to the imaging system. The pulse signal is also monitored with an oscilloscope in order to have a means to verify the pass and fail behaviour prior to integration with the microscope.

The pattern run time is $66 \mu \mathrm{s}$ in this case. The pulse length is therefore set to $33 \mu$ s resulting in a duty cycle of $50 \%$ for a fail. The pixel dwell time is set to $128 \mu \mathrm{s}$, so that there are two test executions per pixel. Therefore an image of $512 \times 512$ pixels can be recorded in about $34 \mathrm{~s}$. The acquisition time is much shorter than previously. The pattern to pattern index time (see method in Fig. 2) of $300 \mathrm{~ms}$ would not allow any of these images even of smaller sizes at reasonable times (e.g. a 128x128 pixel image with $300 \mathrm{~ms}$ per pixel would result in $82 \mathrm{~min}$, but the stability of the laser scanner is only guaranteed within a time of approximately 15 to $20 \mathrm{~min}$ ).

The local illumination changes the PASS/FAIL state of the DUT and thus the observed pulse signal. The SDL images are overlaid with previously made backside images of the die. These overlay images in $\mathrm{x} 5$ and $\mathrm{x} 100$ 
magnification are shown in Fig. 7. The area observed in the overview image corresponds to the area where the activation and internal reading should happen according to the applied addresses during the pattern. The overlay images in x100 magnification allows a layout overlay and a localization of single transistors. With the precise localization of the spots a preparation of the component to this very location is performed because a defect is suspected at this location. After oxide lift-off a polysilicon short is found. A secondary electron microscope (SEM) image of the short is shown in Fig. 8. For this analysis, another three samples have been investigated and the new method proved to be successful on all parts, because a defect was found in all cases.
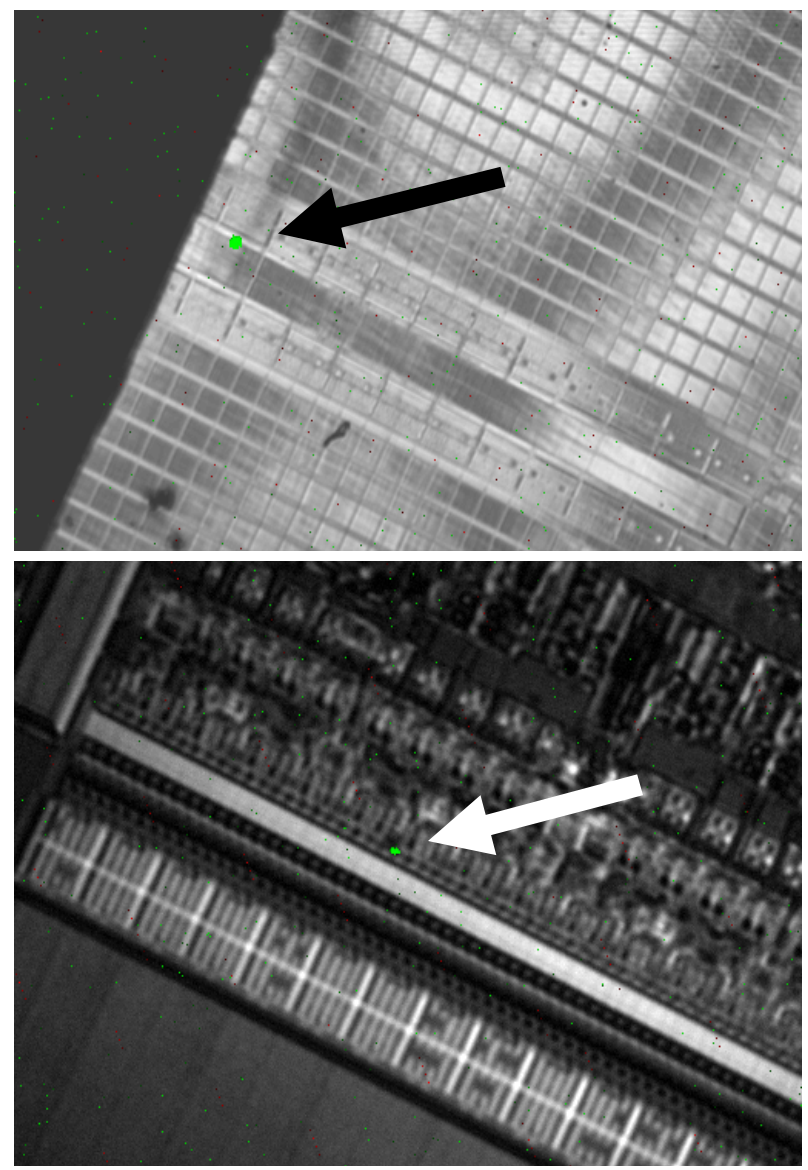

Figure 7: Overview overlay images of the backside and SDL image in $x 5$ and $x 100$ magnification. The arrows mark the position of the observed spot.

In this application case, the defect that is causing the failure is located in the periphery of the DRAM (see also spots in Fig. 7 - the DRAM cell array can be seen in the regular square-like structure in the upper image). Failures caused by defects in the periphery are generally very hard to analyze through bitmapping and knowledgebased FA as it can be seen in [7] for example.

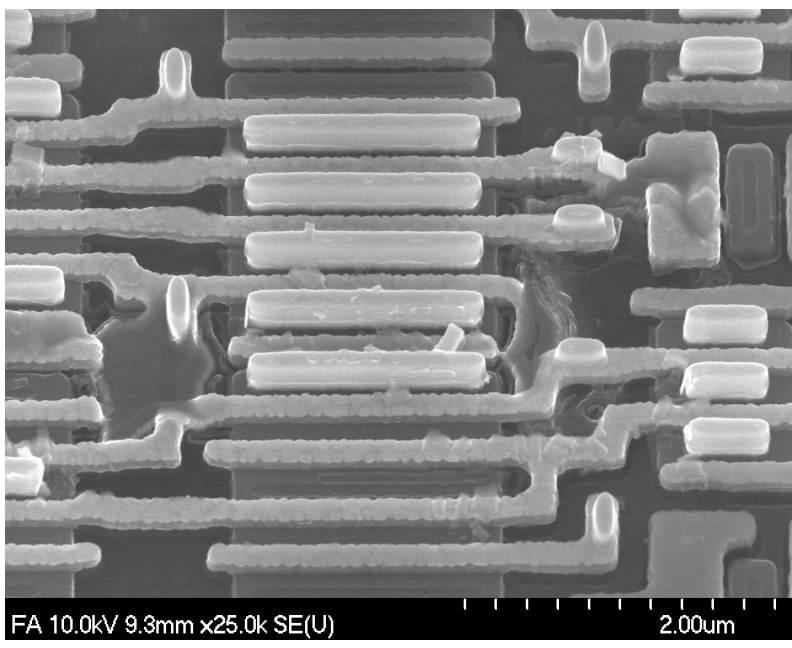

Figure 8: SEM image of gate level shorts after lift-off of the oxide on transistor level.

\section{Conclusions}

A new instrumentation tool was developed for use in conjunction with a Hamamatsu laser scanning microscope for soft defect localization in high speed DRAM memories. The functionality of the tool was verified and a successful application was demonstrated. This method provides a means for localization of temperature dependant defects like shorts and opens in the DRAM periphery where bitmapping is not working very well. The method could also be applied for defect localization within the cell array, but bitmapping seems to be faster in terms of turnaround times.

\section{References}

[1] [1] E.I. Cole Jr. et al., "Resistive Interconnect Localization", ISTFA Proceedings, 43-50 (2001).

[2] M.R. Bruce et al., "Soft Defect Localization (SDL)", ISTFA Proceedings, 21-27 (2002).

[3] F. Beaudoin et al., "Laser Stimulation Applied to Dynamic IC Diagnostics", ISTFA Proceedings, 371-377 (2003).

[4] E. I. Cole Jr., Desk Reference of Microelectronics Failure Analysis, 5th Edition, 415-416 (2005).

[5] Z. Qian, C. Brillert, C. Burmer, M. Grützner, "Lock-In Assisted Soft Defect Localization (LIA-SDL) and its Application In Scan Shift Problems“, ISTFA Proceedings, 128-135 (2005).

[6] M. Versen, D. Diaconescu, J. Touzel, "Soft defect localization technique for design and debug on DRAM devices", ISTFA Proceedings, 426-430 (2006).

[7] M. Versen, D. Diaconescu, J. Touzel, "Case Study and Fault Modelling for Wrong Redundancy Evaluation on DRAM Devices", ISTFA Proceedings, 252-257 (2007). 